\title{
Die Hausärzte sterben aus oder Der moderne Patient
}

\section{Konrad Michel}

Prof. em. Dr. med., Psychiater

Hausärzte, die Dodos und Säbelzahntiger unter uns, zum Aussterben verurteilt [1]. Schlimm. Die Welt hat sich verändert und braucht sie nicht mehr. Der moderne Patient geht direkt in die Comprehensive Sports Clinic. Subito, und mit MRI und Labor vom Hb bis Molybdän.

Ok, der moderne Mensch mag selbstbestimmt, technologie-, internet- und facebookgläubig sein. Als Psychiater behaupte ich, dass die Grundsubstanz die gleiche geblieben ist. Der Mensch sucht nach wie vor Sicherheit, vor allem "in times of calamity" (J. Bowlby, Vater der Bindungstheorie), also in Zeiten der Not. Ich weiss, dass viele Leute heute nicht mehr ihren Hausarzt haben. Leider ist das so. Das heisst aber nicht, dass es die andern nicht mehr gibt. Vor 40 Jahren hörte ich von Freunden, die ein Literaturstudium gewählt hatten, Dialekte würde es in unserem Land in 20 Jahren nicht mehr geben. Man sagte den Tod des Kinos und des gedruckten Buches voraus. Die Realität

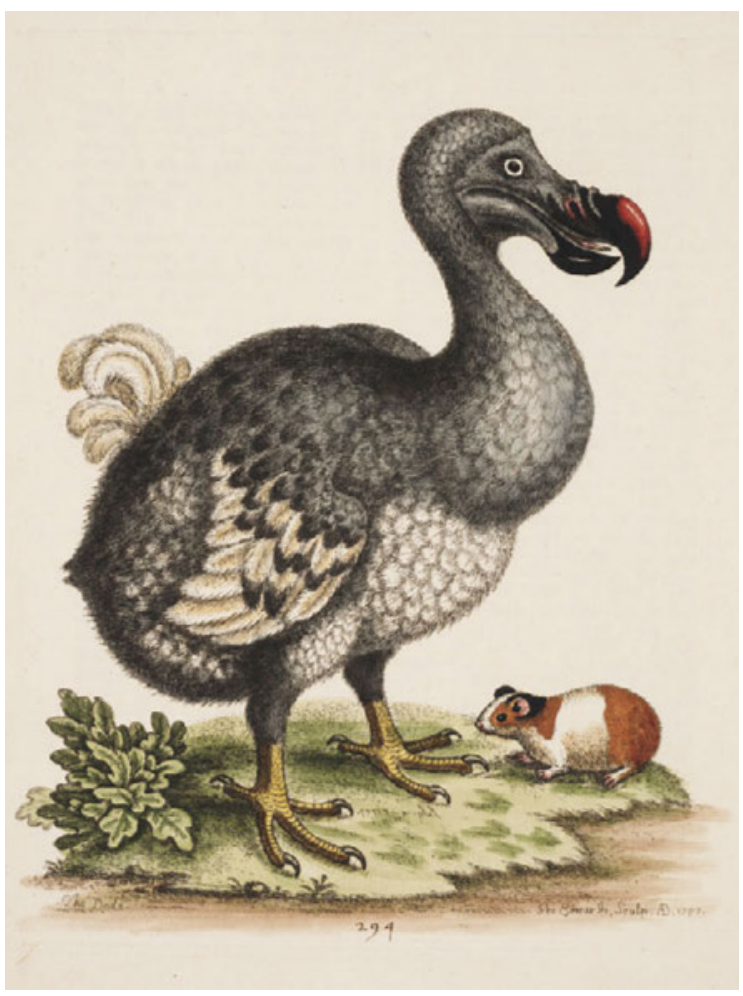

ist, dass der Dialekt neben Frühenglisch, das Kino neben TV und Streaming, das Buch neben eBooks weiter - und gut-existiert.

Liebe Hausärzte, können Sie sich vorstellen, dass Sie neben den Ärztezentren am Bahnhof - mit wechselndem Personal - weiterhin eine wichtige Rolle in der Gesundheitsversorgung spielen? Dass es zwar Patienten gibt, die finden, den Hausarzt brauche es nicht, während andere beim Hausarzt langfristig Sicherheit und Verständnis suchen - und noch einmal andere den

\section{Der Mensch sucht nach wie vor Sicherheit,} vor allem «in times of calamity".

Hausarzt und die Subito Comprehensive Clinic nebeneinander brauchen. Sind wir überzeugt, dass der Bankangestellte, der vom Bike fällt, nicht wiederum beim Hausarzt, der ihn durch die Scheidung begleitet hat, erscheinen wird, wie auch die andern zwei Patientinnen? Ja, man könnte gekränkt sein, wenn die Leute neben dem Dorfladen auch bei Lidl einkaufen, das ist naheliegend. Ich denke, besser ist es, sich auf die veränderte Welt einzustellen.

Der Hausarzt ist der Experte der Arzt-Patienten-Beziehung. Lassen Sie mich etwas dazu sagen. Die gute alte Arzt-Patienten-Beziehung hat etwas mit Vertrauen und Ernst-genommen-Werden zu tun. Vor 30 Jahren erlebte ich das als Patient bei einem Internisten alter Schule. Prof. C. hatte nicht nur immer eine frische Rose auf seinem Schreibtisch, sondern nahm sich Zeit - und notierte Anamnese und Symptome sorgfältig mit der Füllfeder. Dieses wunderbare Gefühl des Aufgehobenseins und der Sicherheit kann ich noch heute jederzeit wachrufen. Das ist nicht etwas von gestern, sondern hochmodern. Im spannenden Buch von Benedetti [2] wird vieles, was zur guten Arzt-Patienten-Beziehung gehört, neurowissenschaftlich belegt. So lässt sich zum Beispiel der Placeboeffekt als Veränderung der neuralen Aktivierung im Gehirn des Patienten zeigen. Empathie ist gut, aber Hoffnung vermitteln ist besser: Die Erwartung auf Linderung der Beschwerden setzt das Belohnungshormon Dopamin frei. Medikamente können ein Vehikel der ärzt- 
lichen Fürsorge, eine Art "token", sein, das der Patient nach Hause nimmt (was so nebenbei für die Selbstdispensation sprechen würde ...). Für Balint war der Arzt selber das "Pharmakon". Eine vertrauensvolle Arzt-Patienten-Beziehung setzt Oxytocin frei

\section{Was beim modernen Patienten dazugekommen ist, ist das Bedürfnis nach Information.}

und inhibiert die Amygdala; dies wird durch körperliche Untersuchung und Berührung gefördert. Wir können natürlich zum Messen des Pulses dem Patienten so ein neues Ding auf die Fingerkuppe stülpen; besser ist es, 20 Sekunden neben den Patienten zu sitzen und den Puls zu palpieren.

Was beim modernen Patienten dazugekommen ist, ist das Bedürfnis nach Information. Das Googeln der Symptome führt in erster Linie zur Verunsicherung. Hier hat der Hausarzt eine Möglichkeit, sich als zuverlässige Informationsquelle zu profilieren. Psychoedukation meint nicht eine dozierende Einwegkommunikation des Arztes, sondern einen gemeinsamen therapeutischen Dialog [3]. Die längerfristige Arzt-PatientenBeziehung schafft die Voraussetzung dazu: eine sichere Vertrauensbasis, einen sicheren Ort. John Bowlby, der Vater der Bindungstheorie, nannte das die Sichere Basis, etwas, was wir nicht nur in der Kindheit, sondern auch als Erwachsene «in times of calamity" brauchen. Es soll mir niemand angeben, dass der moderne Mensch sich so weit emanzipiert hat, dass er das nicht mehr braucht.

Der moderne Arzt schreibt nicht mehr mit dem Füller. Selbstverständlich hat er den PC oder Laptop vor sich. Ist das schlecht für die Arzt-Patienten-Beziehung? Ich glaube nicht. Vielleicht lässt sich das sogar für die therapeutische Beziehung einsetzen. Zum Beispiel lassen sich auf dem Bildschirm dem Patienten relevante Informationen und Abbildungen schnell und effizient zeigen - und ausdrucken (übrigens: Wie wäre es mit einem zweiten, zum Patienten gerichteten Bildschirm?). Wir haben vor Jahren im Rahmen einer Kurztherapie für suizidale Patienten angefangen, eine schriftliche Information («Suizid ist keine überlegte Handlung») abzugeben. Wir waren überrascht, wie viele Patienten spontan berichteten, dass sie diese Information ihren Familienangehörigen zeigten und der Text in der Familie die Runde machte; erstaunlich vor allem auch, wenn man bedenkt, wie viel Information man zum Thema Suizid im Internet findet. Wir stellten aber auch fest, dass Patienten oft Bemerkungen an den Rand kritzelten, was uns bewog, Leerzeilen einzufügen und die Anmerkungen der Patienten beim nächsten Termin zu besprechen.
Liesse sich so etwas nicht auf die Hausarztpraxis übertragen? Wir wissen ja, dass Patienten nach einem Arztbesuch kaum zuverlässig wiedergeben können, was der Arzt gesagt hat. In der modernen Informationsgesellschaft ist die überzeugende - schriftliche oder digitale - Information von vertrauenswürdiger Stelle entscheidend. Wenn es um Gesundheitsprobleme geht, reicht es eben nicht, die Symptome zu googeln. Die Information vom Arzt muss klar, überzeugend, gut verständlich und wenn möglich persönlich sein. Neu ist das ja nicht - bei chronischen Krankheiten wie Diabetes ist das schon lange Standard. Es könnte hilfreich sein, wenn Fachgesellschaften dem Hausarzt Patienteninformationen elektronisch zur Verfügung stellten, die er allenfalls noch "personalisieren" kann. Es macht einen grossen Unterschied, ob der Patient eine "persönliche» schriftliche Information seines Arztes oder eine unpersönliche Hochglanzinformation einer Pharmafirma bekommt. Vielleicht haben Sie auch schon festgestellt, dass Patienten im Wartezimmer vom Arzt aufgelegte Gesundheitsinformationen der Schweizer Illustrierten vorziehen.

\section{In der modernen Informationsgesellschaft ist} die überzeugende Information von vertrauenswürdiger Stelle entscheidend.

«The ritual of the therapeutic act per se has a profound impact on the patient's brain, and every effort should be made by the clinician to enhance it» (Benedetti [1], S. 182). In diesem Sinn möchte ich beliebt machen, sich auf die Bedeutung der Arzt-Patienten-Beziehung in einer veränderten Welt zu besinnen. Das ist die Chance für den Hausarzt. Wir Psychiater wollen nicht die einzigen bleiben, die die therapeutische Beziehung pflegen.

Übrigens: Auch der mit dem Bildschirm arbeitende moderne Hausarzt könnte zum Beispiel das Rezept mit der Füllfeder schreiben ...

\section{Bildnachweis}

Von George Edwards - Edwards, George, 1694-1773. Gleanings of natural history, exhibiting figures of quadrupeds, birds, insects, etc (1760), plate 294. London Royal College of Physicians. (from http://images. library.wisc.edu/DLDecArts/EFacs/NatHistEd1/NatHistEd06/M/0194. jpg Digital Library for the Decorative, Gemeinfrei, https://commons. wikimedia.org/w/index.php?curid=13027169)

\section{Literatur}

1 Oertle D. Die Hausärzte sterben aus - na und? Schweiz Ärztezeitung. 2016;97(51-52):1804-5.

2 Benedetti F. The Patient's Brain: The Neuroscience Behind the Doctor-Patient Relationship. Oxford University Press 2010. 304 pp. ISBN 9780199579518

3 Colom F. Keeping therapies simple: psychoeducation in the prevention of relapse in affective disorders. The British Journal of Psychiatry. Apr 2011;198(5):338-40. 